\title{
Definitive screening for accelerated Taxol biosynthetic pathway optimization and scale up in Saccharomyces cerevisiae cell factories
}

\author{
Laura Walls ${ }^{1}$, José Martinez ${ }^{2}$, E. Antonio del Rio Chanona ${ }^{3}$, and Leonardo Rios Solis ${ }^{1}$ \\ ${ }^{1}$ The University of Edinburgh School of Engineering \\ ${ }^{2}$ Technical University of Denmark \\ ${ }^{3}$ Imperial College London Department of Chemical Engineering
}

September 24, 2021

\begin{abstract}
Recent technological advancements in synthetic and systems biology have enabled the construction of microbial cell factories expressing diverse heterologous pathways in unprecedentedly short time scales. However, the translation of such laboratory scale breakthroughs to industrial bioprocesses remains a major bottleneck. In this study, an accelerated bioprocess development approach was employed to optimize the biosynthetic pathway of the blockbuster chemotherapy drug, Taxol. Statistical design of experiments approaches were coupled with an industrially relevant high-throughput microbioreactor system to optimize production of key Taxol intermediates, Taxadien- $5 \alpha$-ol and Taxadien- $5 \alpha$-yl-acetate, in engineered yeast cell factories. The optimal factor combination was determined via data driven statistical modelling and validated in $1 \mathrm{~L}$ bioreactors leading to a 2.1-fold improvement in taxane production compared to a typical defined media. Elucidation and mitigation of a nutrient limitation enhanced product titers a further two-fold and titers of the critical Taxol precursors, Taxadien- $5 \alpha$-ol and Taxadien$5 \alpha$-yl-acetate were improved to 34 and $11 \mathrm{mg} / \mathrm{L}$, representing a three-fold improvement compared to the highest literature titers in S. cerevisiae. Comparable titers were obtained when the process was scaled up a further five-fold using $5 \mathrm{~L}$ bioreactors. The results of this study highlight the benefits of a holistic design of experiments guided approach to expedite early stage bioprocess development.
\end{abstract}

\section{Hosted file}

Manuscript for Submission to Biotechnology Journal.docx available at https://authorea.com/ users/331623/articles/538329-definitive-screening-for-accelerated-taxol-biosyntheticpathway-optimization-and-scale-up-in-saccharomyces-cerevisiae-cell-factories 


\section{A)}

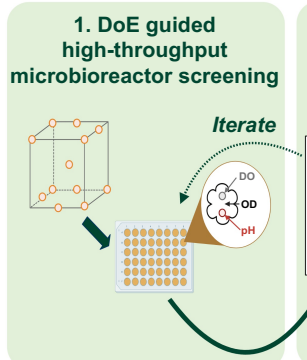

2. Statistical analysis
and model

development

3. Validation \& further optimisation at $1 \mathrm{~L}$ scale up

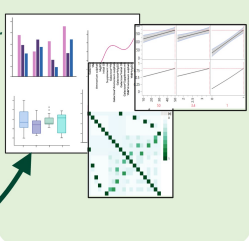

dite
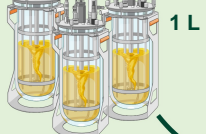

$5 \mathrm{~L}$

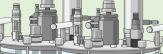

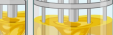

B)

LRS5 \& LRS6

LRS6 only

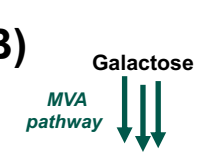

Iso-Taxadiene

GGPP
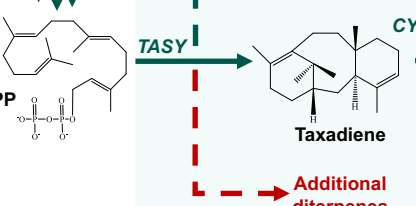

CYP725A4

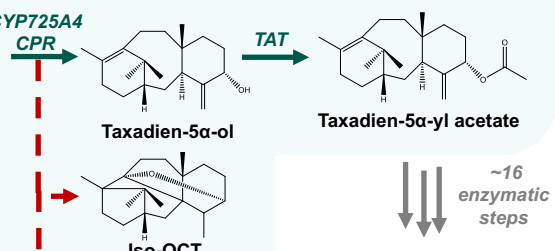

Taxanes

I ISO-OCT

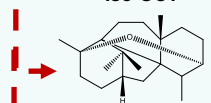

I $\begin{aligned} & \text { OCT } \\ & \text { I Additional }\end{aligned}$

$I \rightarrow \begin{gathered}\text { Additional } \\ \text { oxygenated } \\ \text { diterpenes }\end{gathered}$

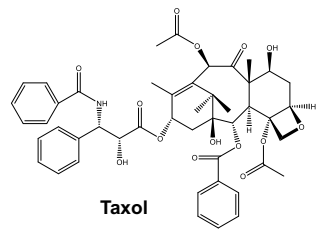


figures/Figure-2/Figure-2-eps-converted-to.pdf 
figures/Figure-3/Figure-3-eps-converted-to.pdf 
figures/Figure-4/Figure-4-eps-converted-to.pdf 
figures/Figure-5/Figure-5-eps-converted-to.pdf 
figures/Figure-6/Figure-6-eps-converted-to.pdf 
figures/Figure-7/Figure-7-eps-converted-to.pdf 